González Martínez, Juan; Espuny Vidal, Cinta; de Cid Ibeas, M. ${ }^{a}$ José; Gisbert Cervera, Mercè (2012). INCOTICESO. Cómo autoevaluar y diagnosticar la competencia digital en la Escuela 2.0. Revista de Investigación Educativa, 30 (2), 287-302.

\title{
INCOTIC-ESO. CÓMO AUTOEVALUARY DIAGNOSTICAR LA COMPETENCIA DIGITAL EN LA ESCUELA 2.0
}

\author{
Juan González Martínez' \\ Cinta Espuny Vidal \\ M. ${ }^{a}$ José de Cid Ibeas \\ Mercè Gisbert Cervera \\ Universitat Rovira iVirgili \\ Facultat de Ciències de l'Educació i Psicologia
}

\section{RESUMEN}

La implementación de la Escuela 2.0 pivota, en parte, sobre la competencia digital del alumnado de Ciclo Superior de Educación Primaria o Primer Ciclo de Educación Secundaria. Sin embargo, esta competencia tan importante en la Sociedad del Conocimiento no está suficientemente consolidada por el alumnado. Para ello, se ha diseñado INCOTIC-ESO, una herramienta diseñada para realizar una evaluación autodiagnóstica de la competencia digital que nos permitirá ajustar la planificación de la docencia de esta competencia cuando sea necesario. El objetivo de este artículo es presentar el proceso de diseño, validación y fiabilización de esta herramienta así como los primeros datos extraídos de su aplicación a un grupo piloto.

Palabras clave: Tecnología digital, evaluación, educación secundaria, instrumentos, TIC.

\footnotetext{
Correspondencia:

Juan González Martínez. E-mail: juan.gonzalezm@urv.cat, cinta.espuny@urv.cat, mcid3@xtec.cat, merce.gisbert@urv.cat

1 Los Dres. Juan González, Cinta Espuny y Mercè Gisbert pertenecen al Grupo de Investigación ARGET (Applied Research Group in Education and Technology), de la Universitat Rovira i Virgili, cuyo identificador es 2009 SGR 596. Asimismo, esta investigación se ha llevado a término dentro del proyecto Simul@: Evaluación de un Entorno Tecnológico de Simulación para el Aprendizaje de Competencias Transversales en la Universidad, con referencia EDU2008-01479, del plan nacional de I+D+i del Ministerio de Educación y Ciencia.
} 


\title{
INCOTIC-ESO. SELF-ASSESSMENT AND DIAGNOSIS OF DIGITAL COMPETENCE AT "SCHOOL 2.0"
}

\begin{abstract}
Spanish Program «Escuela 2.0» (one student, one laptop) in Compulsory Education is partially based on students' digital competence, which is of paramount importance in today's Knowledge Society. Yet, many students have not fully developed this competence. INCOTICESO is a tool designed for self-assessing the digital competence, which will enable adjusting the teaching of this competence when necessary. The aim of this paper is to present the design, validation and reliability process of this tool, as well as the first data after its implementation on a pilot group.
\end{abstract}

Keywords: digital technology, assessment, secondary education, tools, ICT.

\section{INTRODUCCIÓN}

La apuesta decisiva de nuestras instituciones educativas - en este caso, el Ministerio de Educación - por explotar de modo intensivo en las aulas las Tecnologías de la Información y la Comunicación, como sabemos, ha venido a materializarse en las directrices de la llamada Escuela 2.0. Esta apuesta, en general, comparte que dicha materialización considere el desembarco de portátiles o microportátiles en las aulas, insertos ya como un recurso de primer orden en la práctica cotidiana. Amén de innumerables cuestiones pedagógicas y organizativas (González Martínez 2010b), es indudable que esta revolución en nuestras aulas de educación obligatoria pivota sobre una competencia fundamental, la digital, cuya naturaleza y cuyos umbrales de afianzamiento se están definiendo caso de modo paralelo a la práctica. Nuestro alumnado -y nuestro profesorado también, sin ninguna duda- deberá ser competente digitalmente para afrontar el aprendizaje del modo como se concibe este bajo el paraguas de la Escuela 2.0; y por ello, también sin ninguna duda, deberemos estar atentos, vigilantes, ante la competencia digital de ese alumnado. Por tanto, deberemos plantearnos cuál es su nivel inicial, cómo planificamos su adquisición y cómo acreditamos, al finalizar los estudios secundarios obligatorios - ESO, en nuestro caso-, que nuestro alumnado ha alcanzado un nivel suficiente, que le permite convertirse en un ciudadano competente, también desde el punto de vista digital.

A buena parte de estas cuestiones se dedica nuestra propuesta presente: a desgranar el concepto de competencia digital, en el contexto del programa eduCAT 1x1 (concreción catalana para la Educación Secundaria Obligatoria de la estatal Escuela 2.0), y a diseñar una primera herramienta con que realizar una autoevaluación diagnóstica de la competencia digital para nuestro alumnado - INCOTIC-ESO-, que sirva de partida y nos permita planificar su docencia a lo largo de toda la etapa educativa. 


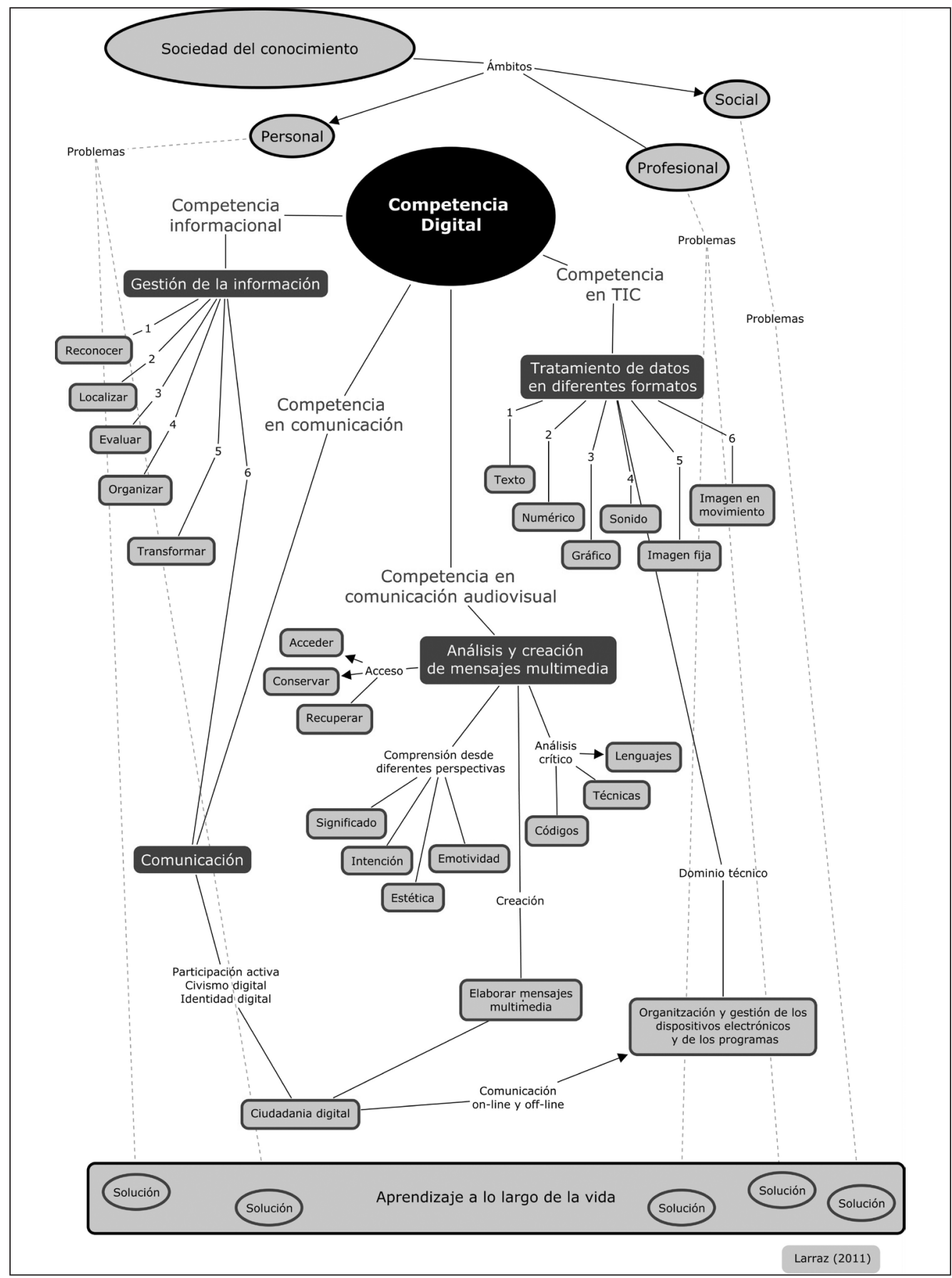

IMAGEN 1

LA COMPETENCIA DIGITAL Y EL APRENDIZAJE A LO LARGO DE LA VIDA (LARRAZ, 2011) 


\section{ACERCA DE LA COMPETENCIA DIGITAL}

Si nos centramos ahora de manera específica en la competencia digital, convendremos que supone la adquisición de conocimientos, destrezas y actitudes que tienen que ver con el uso elemental del hardware de los ordenadores, sus sistemas operativos como gestores del hardware, el software como herramienta de trabajo, de comunicación off-line y de comunicación on-line; y, por extensión de la competencia de gestión de la información, todo aquel uso de las TIC que tenga que ver en los procesos de localización, acceso, obtención, selección, gestión y uso de esta información (URV 2009). A lo que habría que añadir su utilización en el momento preciso, de modo eficaz y eficiente.

En definitiva, entendemos por competencia digital la conjunción de lo que muchos autores entienden por competencia TIC y competencia informacional. En la sociedad del conocimiento no tiene sentido hablar solo de herramientas para el almacenaje, acceso y recuperación de la información, sino que debemos trabajar, también, las habilidades y las destrezas necesarias para usar adecuadamente esta información y transformarla después en conocimiento, con el objetivo final de compartirlo. Es, en parte, el concepto de competencia digital propuesto por Vivancos (2008), que Larraz (2011) resume en esta imagen, aplicada a la competencia digital y a su incidencia en el aprendizaje a lo largo de la vida.

\section{NUESTRO ALUMNADO DE ESO: ¿NATIVOS DIGITALES?}

A menudo, acaso en exceso, partimos de la constatación de que nuestro alumnado pertenece de pleno a lo que, en teoría, se ha venido conociendo como nativos digitales (Pensky, 2001), o lo que unos años más tarde Oblinger \& Oblinger (2005) denominaron la Generación. NET (que también se conoce a menudo como Generación Messenger o Generación Google). Con todo, y sin entrar ahora en una discusión acerca del riesgo que entraña afirmar tal cuestión, estamos convencidos de que debemos plantearnos qué implicaciones tiene ello en términos de formación y de aprendizaje: ¿Pertenecer a dicha generación ya determina per se que los estudiantes han adquirido la competencia digital, entendida como decíamos como la suma de la competencia TIC y la competencia informacional? Y, suponiendo que ello pueda afirmarse, ¿en qué grado la han adquirido? $\mathrm{O}$, lo que es más, ¿cómo podemos conseguir evidencias de ello? $\mathrm{Y}$, por supuesto, todo ello con el fin de que la formación que vamos a impartirles se adecue a sus necesidades $y$, por tanto, se maximicen los resultados de aprendizaje, que es nuestro fin último.

Ni todas estas preguntas tienen una respuesta evidente ni tenemos datos empíricos de que estas nuevas generaciones, gracias a las TIC, tengan más y mejores habilidades de aprender. Y de ello se desprende con facilidad que, para poder fundamentar estas aseveraciones, será imprescindible desarrollar estudios e investigaciones que nos permitan confirmar o refutar estas afirmaciones. Para ello, necesitamos instrumentos que nos permitan recoger datos, de una manera sistemática y fiable, de tal modo que con ellos estamos en disposición de demostrar cuáles son las características reales de todos los estudiantes que pasan por nuestras manos, por lo que a competencia digital se refiere. En principio, es evidente que los estudiantes actuales están inmersos en un 
mundo tecnológico y que los dispositivos móviles se han convertido en una herramienta inherente a su propia vida cotidiana. En efecto, seguramente tienen muchas potencialidades comunicativas, pero no tenemos suficientes evidencias para poder asegurar que además lea ayudan a formarse mejor (Bullen 2009).

En este contexto de la necesidad de recabar datos e investigar todo el proceso de generalización de las TIC en la docencia, debemos enmarcar la herramienta que presentamos en este artículo, INCOTIC-ESO: un cuestionario cuyo propósito es recoger información acerca de la autoconcepción de nivel de competencia digital propio con el que llegan los estudiantes a primer curso de la ESO en nuestro caso (y fácilmente adaptable cuando el propósito sea aplicarlo al ciclo superior de Educación Primaria, en el caso de aquellas comunidades autónomas en que la Escuela 2.0 haya empezado a implantarse en esa etapa). Sin duda, esta información nos ayudará a generar unos procesos de formación y acreditación adecuados.

\section{LA CREACIÓN DE LA HERRAMIENTA: INCOTIC-ESO}

La competencia digital se ha convertido en algo imprescindible para afrontar los retos de la vida cotidiana de la ciudadanía, a la vez que resulta fundamental para el desarrollo académico y posteriormente profesional de cualquier estudiante; y por ello aparecen en lugar preeminente en la nómina de destrezas no terminales o específicas cuyo conocimiento deberá acreditar el alumnado en el momento de la finalización de su Educación Secundaria Obligatoria. No en vano, la adquisición de la competencia digital supone una poderosa herramienta para cualquier futuro profesional $y$, por ello, cualquier etapa educativa debe considerarla como uno de los elementos sustanciales para la adquisición de la ciudadanía por parte de su alumnado. Tal y como lo recogen Aguaded, Guzmán y Pavón (2010), «las TIC [...] han supuesto un gran enriquecimiento de la vida académica y científica, no solo por su utilización como herramientas en los procesos de investigación y producción científica, sino también como medio de comunicación». Y, por tanto, de ellas se aprovecha -y no poco- el cambio metodológico que entrañan la Escuela 2.0, en la enseñanza obligatoria, y la convergencia hacia el EEES, en los estudios universitarios (De Pablos 2007).

Sin embargo, la docencia de la competencia digital entraña un reto que debemos asumir: cómo la planificamos, cómo la evaluamos, cómo diseñamos el proceso de formación en esta competencia a lo largo de toda una etapa educativa y, lo que es más importante, cómo recogemos evidencias del grado de adquisición de esta competencia por parte del estudiantado que llega a los primeros cursos de la Educación Secundaria Obligatoria. Por esta razón, nos hemos propuesto el diseño y desarrollo de una herramienta para la autoevaluación diagnóstica de la competencia digital en la ESO: Inventario de Competencias TIC, INCOTIC-ESO.

La autoevaluación diagnóstica de la competencia digital, previa a la planificación concreta de la docencia, persigue conocer cuáles son los conocimientos previos que tienen adquiridos los estudiantes, desde su punto de vista, al iniciar sus estudios secundarios obligatorios. Así pues, los objetivos generales de la herramienta son:

1. Obtener información sistematizada de la percepción que el estudiantado tiene de su nivel de competencia digital. 
2. Realizar una autoevaluación diagnóstica de los estudiantes de primer curso de ESO en cuanto a su nivel de competencia digital.

3. Servir de pauta para la organización y para el diseño de la oferta formativa posterior.

Somos conscientes de las limitaciones que puede tener un proceso de autoevaluación en cuanto a la correcta percepción del propio grado de su adquisición por parte de cada sujeto; con todo, creemos que puede constituir un primer aporte de información fundamental que sirva como guía al profesorado y a cada uno de los centros escolares a la hora de diseñar y desarrollar acciones formativas encaminadas a la trabajar todos estos aspectos con el alumnado.

\section{I. Génesis del cuestionario de diagnóstico}

INCOTIC-ESO supone la adaptación y reconcepción de una herramienta ya creada, INCOTIC-Grado, destinada a un mismo afán de radiografiar la competencia digital del alumnado, en este caso universitario, al inicio de sus estudios universitarios, y cuyo diseño y validación pueden ampliarse en González Martínez (2010a) y Gisbert Cervera (2011). Aquella herramienta, a su vez, parte de un recorrido que viene de atrás, y cuyas pistas pueden seguirse en las diversas adaptaciones de Marqués (2006) y Lleixà (2008), en los que con diferentes objetivos se pretendía conocer la competencia digital del alumnado universitario, al servicio de su aprendizaje.

El diseño de una herramienta parte de la revisión de la literatura acerca de la concepción de la competencia digital, partiendo de un terno de fuentes y ángulos de perspectiva. Por una parte, fue necesario partir de los diferentes estudios y experiencias que han abordado la reflexión y el diseño de herramientas para evaluar la competencia digital (véase, de nuevo, González Martínez, 2010a). Además, tuvimos en cuenta aportaciones como Vivancos (2008), quien señala que la competencia digital, en sus nuevas formulaciones, «tal y como se especifica en los Reales Decretos de las enseñanzas mínimas que despliega la Ley Orgánica 2/2006, de 3 de mayo, de Educación, se formula como un concepto integrador que acoge, en buena medida, los postulados de tres alfabetizaciones: la informacional, la audiovisual y la informática o TIC».

En segundo lugar, fue imperativo atender a lo establecido en la Acreditación de Competencias en Tecnologías de la Información y la Comunicación (ACTIC), regulada por el decreto 89/2009, de 9 de junio, del Departamento de Gobernación y Administraciones Públicas de la Generalitat de Catalunya. ACTIC es un certificado acreditativo de la competencia digital que permite que a partir de los 16 años, edad que finalizan la educación secundaria obligatoria, se pueda, mediante la superación de una prueba, acreditar el correspondiente nivel competencial TIC. Cuenta con tres niveles: básico, mediano o avanzado, y se ha diseñado pensando en su validez y pertinencia tanto en el entorno empresarial y como en el administrativo.

Y, cómo no podía ser menos, por último, he sido imprescindible atender a la definición y la catalogación de las competencias que la Ley Orgánica de Educación (LOE, 2/2006 de 3 de mayo) especifica; y, más concretamente, a la competencia digital, entendida como la suma de tres competencias. 
Triangulada la información obtenida desde las diferentes partes: por una parte, la LOE, por otra aportaciones de autores, y la ACTIC, pasamos a aglutinar en diferentes etiquetas los diferentes conceptos. $Y$, a su vez, establecimos los pilares de la competencia digital y, con ellos, las diferentes rúbricas que se desprenden de todo ello.

En este proceso, una de las mayores dificultades recae en catalogar las diferentes rúbricas que engloban la competencia digital. Para ello fue necesario, por una parte, entender los diferentes elementos que engloba la competencia digital, contextualizarlo en la etapa educativa correspondiente, Educación Secundaria Obligatoria (ESO), edad comprendida de los 12 a los 16 años; y, más concretamente, centrarnos en la edad de los usuarios de esa herramienta de autodiagóstico, alumnado de 12-13 años.

Otra de las dificultades era determinar un lenguaje óptimo para el alumnado y tener presente el objetivo, radiografiar la competencia digital del alumnado de primero de ESO al iniciar su curso mediante un cuestionario sobre la autopercepción de su competencia digital, con el fin de poder conocer el punto de partida de dicho alumnado, y poder acompañarle en esta etapa educativa con el fin de que éstos adquieran dicha competencia. Esos datos, a su vez, nos permitirán diseñar acciones formativas al respecto, durante los cursos que permanezca en la etapa, y poder establecer comparaciones grupales e individuales a la vez, siempre respetando el anonimato y con la finalidad de una investigación, basada en principios educativos y éticos.

Durante el curso 2009-2010, con las primeras acciones para la convergencia de los centros públicos de educación secundaria obligatoria para la llegada del programa eduCAT $1 x 1$ (concreción de la Escuela 2.0 del resto del Estado), desde la URV, se procedió a todo este proceso de creación de la herramienta INCOTIC-ESO, como respuesta a la necesidad de compartir desde la universidad toda esta revolución pedagógica y colaborar, en la medida de lo posible, en ese proceso. En este sentido, y a modo de resumen, las acciones que nos han llevado a la construcción de la herramienta han sido las siguientes:

1. Revisar el concepto de competencia digital, integrando en la definición la disponibilidad de recursos TIC, sus usos, su formación; ajustar su valoración en relación con los niveles demandados por la correspondiente legislación educativa para Educación Secundaria Obligatoria.

2. Adaptarlo al formato digital, basado en la concepción y las herramientas de la Web 2.0.

3. Realizar una primera aplicación a una muestra, con estudiantes voluntarios, de la población matriculada en segundo curso de ESO en el INS Joaquim Bau (Tortosa), durante el mes de junio de 2010 [ $\mathrm{N}=50]$ que ha servido de pilotaje para la validación y fiabilización de INCOTIC-ESO.

\subsection{La versión digital del cuestionario}

El estudiantado accede al cuestionario por medio de la utilidad Spreadsheets ${ }^{\odot}$ de Google, con la intención de agilizar no sólo su respuesta, sino también el vaciado y la sistematización de los datos. Por tanto, INCOTIC-ESO cumple, como herramienta de autodiagnóstico de la competencia digital, con la exigencia de integración en la interfaz de la Web 2.0 y las ventajas que ello conlleva: 
1. Facilita posteriores modificaciones y adaptaciones.

2. Permite una variada tipología de opciones de preguntas y también de respuestas.

3. Genera de manera automática un primer tratamiento de estadísticos básicos que puede ayudar a definir con más exactitud hacia dónde ampliar posteriores análisis más pormenorizados.

4. Ofrece la posibilidad de exportar los datos a una hoja de cálculo, lo que favorece la realización de análisis en más profundidad por medio de paquetes informáticos estadísticos, como PASW o Statgraphics Centurión, por citar algún ejemplo.

5. En contraposición a los cuestionarios orales o en papel, facilita y dinamiza la acción de la respuesta.

6. El entorno GoogleDocs permite compartir, modificar y distribuir el cuestionario con gran facilidad.

Por último, haremos referencia al proceso mismo de distribución del cuestionario durante el presente pilotaje, que ha sido alojado en el espacio Moodle del centro, para que todos los alumnos que participaron en el pilotaje tuvieran fácil acceso a él, aunque puede ser alojado en cualquier entorno virtual de enseñanza-aprendizaje (EVEA).

\subsection{Estructura del cuestionario}

Para la reelaboración de la herramienta, como ya hemos mencionado, hemos partido de LOE, de los estándares de ACTIC, así como de las reflexiones generales de Storey (2002) acerca de la usabilidad de las herramientas TIC; y también de las consideraciones de Esteve (2009) en referencia a la revolución pedagógica que entraña el proceso de convergencia europeo y las TIC, que en última instancia puede servir de espejo en el que acabe mirándose la educación secundaria obligatoria.

En un principio, consideramos que el cuestionario debía ser anónimo, para facilitar la necesaria sinceridad en las respuestas del alumnado. Sin embargo, en un segundo momento $-\mathrm{y}$ así ha quedado recogido finalmente- determinamos codificarlo por medio de claves que no atentan contra el anonimato de los informantes, pero que sí nos permitirán, llegado el caso, realizar un estudio y un análisis longitudinales de la evolución de los estudiantes a lo largo de toda la ESO. Esta codificación, en definitiva, nos permitirá conocer cuál ha sido la evolución por lo que respecta a competencia nuclear digital informante por informante, comparando el cuestionario inicial, el seguimiento y la evaluación final).

Pasamos, a continuación, a realizar una descripción más detallada del contenido del cuestionario. La primera parte de la herramienta está organizada en tres secciones, como se recoge en la Imagen 2.

Sección A: recoge los datos de identificación con los que estableceremos las primeras distinciones en función de los factores individuales de cada informante (edad, género, centro de estudios y naturaleza, centro de procedencia y primeras experiencias digitales). Es importante conocer en qué medida variables como el sexo, la tipología de centro o el centro de procedencia tienen relevancia en las actitudes y competencias de partida del alumnado.

Sección B: Es fundamental la información que nos puedan facilitar respecto al acceso a los recursos digitales (en esencia, ordenador y conexión a Internet, tanto en el aula 


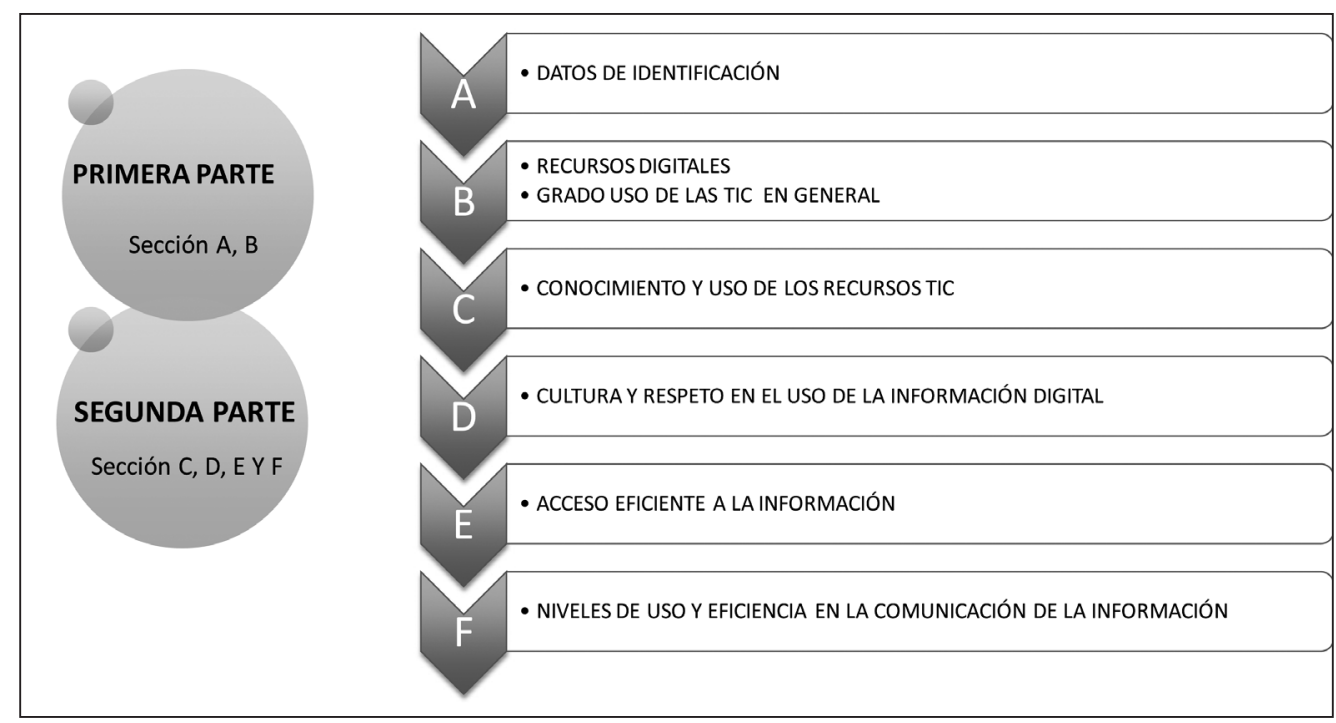

IMAGEN 2

PARTES DEL CUESTIONARIO INCOTIC-ESO

como en el domicilio, y también teléfono móvil) y al lugar donde cotidianamente se produce ese acceso. Por otro lado, nos interesa conocer, especialmente, cuál es el grado real de uso de las TIC en general, no sólo en el contexto académico universitario (usos generales y específicos, tiempo promedio y frecuencia, aportes fundamentales de las TIC).

La segunda parte de nuestro cuestionario nos proporciona los datos que nos permitirán valorar la competencia inicial de nuestro alumnado en TIC. Pasamos a detallar, a continuación, el contenido de cada una de las secciones de esta parte:

Sección C: Referida al conocimiento y uso de los recursos TIC. En la primera parte, abordamos la parte más relacionada con el conocimiento de procesos y recursos generales; en la segunda parte, nos enfrentamos al conocimiento y al uso específico de software altamente rentable en las actividades de aprendizaje; y, en la tercera y última parte de esta sección, nos centramos en cuestiones más relacionadas con la alfabetización tecnológica.

Sección D: Esta sección está dedicada a registrar la cultura y el respeto en el uso de la información digital.

Sección E: Consagrada a sondear el acceso eficiente a la información.

Sección F: Sondea los niveles de uso y eficiencia en la comunicación de la información.

Una vez referidas, en síntesis, la exposición del contenido y de la estructura de la herramienta, pasamos a exponer y valorar cuál ha sido el proceso de pilotaje de la herramienta para valorar su validez y fiabilidad. 


\subsection{Pilotaje, validación y fiabilización de la herramienta}

Para poder asegurar que la herramienta mide, exactamente, aquellas variables que nos planteamos como objetivos a la hora de su diseño es imprescindible someterla a un proceso de validación y fiabilización.

El proceso de validación ha tenido cuatro grandes fases:

- Fase 1: Someter la versión revisada del cuestionario a criterio de un experto TIC experto en competencia digital antes de utilizar el criterio de jueces propiamente dicho. Esta primera acción de contraste nos permitió perfilar tanto el contenido como la redacción utilizada en las diferentes secciones de la herramienta.

- Fase 2: Se distribuyó una primera versión de INCOTIC-ESO a un grupo reducido de estudiantes $(\mathrm{N}=8)$ para perfilar temas de redacción y cuestiones semánticas que aseguraran la correcta comprensión de todos los conceptos y del sentido de las preguntas que se realizan. Tras ello, se analizaron los procesos de traducción (hasta asegurarnos de que solo un porcentaje residual de los informantes tenía problemas de comprensión) y retrotraducción (en este caso, hasta que tuvimos garantías de que solo ese mismo porcentaje mínimo entendía en el cuestionario algo diferente de su propósito).

- Fase 3: Un panel de expertos (siete miembros del ARGET, Applied Research Group in Education \& Technology) revisó y analizó el contenido y la estructura del cuestionario, atendiendo especialmente a cuestiones de orden y con un interés especial puesto en evitar la acción de los posibles sesgos inherentes a los cuestionarios autoadministrados (de tendencia central, de deseabilidad social, de aprendizaje o proximidad, y lógico), por medio de la distribución de las preguntas dentro del cuestionario y de su redacción.

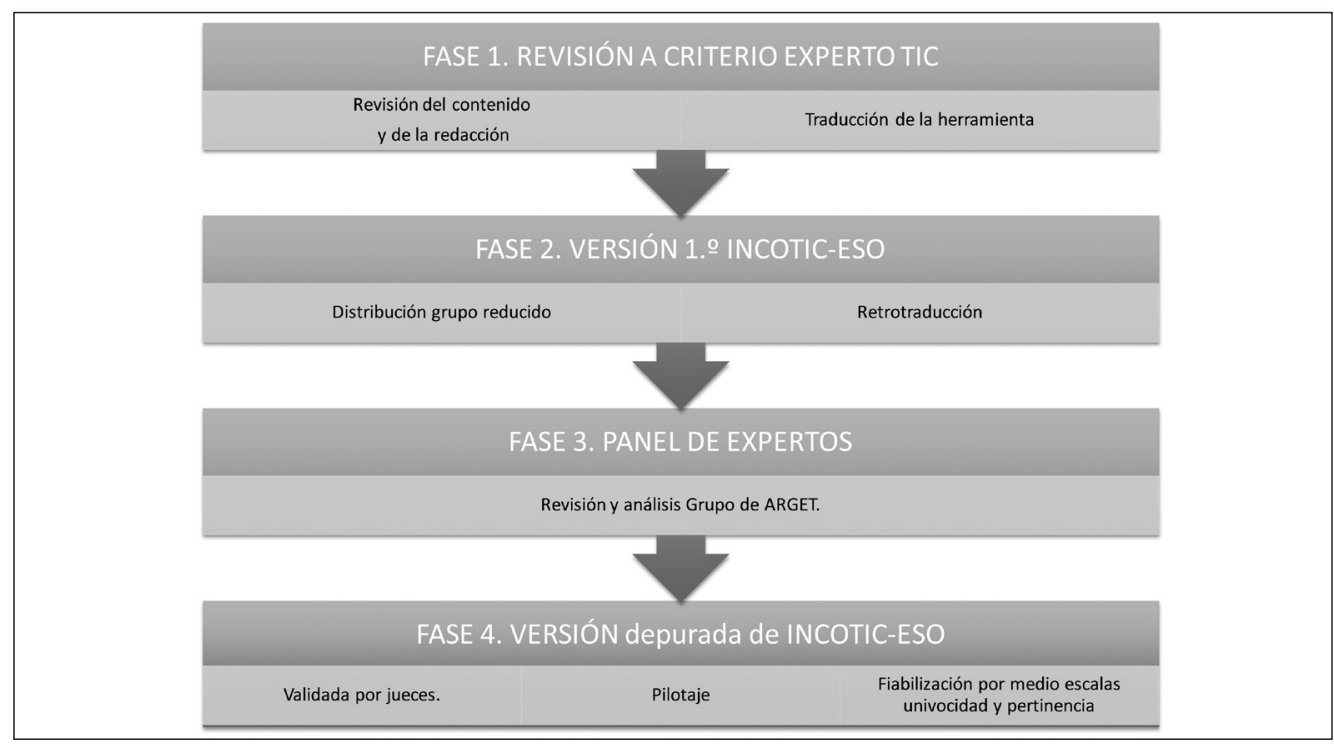

IMAGEN 3

FASES DEL PROCESO DE VALIDACIÓN Y FIABILIZACIÓN DE INCOTIC-ESO 
- Fase 4: La versión ya «depurada» de INCOTIC-ESO fue validada por jueces (5) antes de proceder al pilotaje con el que hemos fiabilizado los datos, por medio de las correspondientes escalas de univocidad y pertinencia.

Resumido en forma de esquema, el proceso que hemos ido siguiendo constó de las siguientes acciones, entre las cuales se contienen las cuatro fases que acabamos de referir:

Con los datos obtenidos en el proceso de pilotaje de la herramienta, en el que participaron 50 sujetos, realizamos un primer análisis de los datos obtenidos, aparte de realizar con ellos las pruebas de fiabilidad correspondientes.

En primer lugar, al agrupar los diferentes ítems que componen cada una de las partes del cuestionario, podemos obtener índices que nos facilitarán los posteriores análisis (todos ellos con un rango de 1 a 5, en la primera parte del cuestionario, y de 1 a 4 en la segunda). Así por ejemplo, por lo que respecta a las variables por medir contenidas en la primera parte del cuestionario, referentes al uso de las TIC, contamos con el Índice de uso; y, en la segunda parte, podemos atender al Índice de Competencia Digital (INCOTIC), al Índice de Hadware, al Índice de Software, al Índice de Alfabetización Tecnológica, al Índice de Cultura Digital, al Índice de Eficiencia en el Acceso a la Información o el Índice de Eficiencia Comunicativa.

Así, por ejemplo, para nuestros sujetos del pilotaje, encontraríamos los siguientes promedios:

TABLA 1

INDICADORES GENERALES DEL PILOTAJE

\begin{tabular}{|l|c|c|c|}
\hline & N & Media & Desviación típica \\
\hline INCOTIC & 50 & 2,8530 &, 44962 \\
\hline Índ. de Recursos & 50 & 2,8620 &, 54235 \\
\hline Índ. de Software & 50 & 2,7380 &, 50149 \\
\hline Índ. de Alfabetización Tecnológica & 50 & 2,7410 &, 69029 \\
\hline Índ. de Cultura Digital & 50 & 3,1467 &, 55751 \\
\hline Índ. de Eficiencia en el Acceso a la Información & 50 & 2,9433 &, 51744 \\
\hline Índ. de Eficiencia Comunicativa & 49 & 2,6837 &, 64367 \\
\hline
\end{tabular}

La inclusión en el cuestionario de las diferentes variables independientes nos permite rastrear las posibles diferencias que puedan existir entre subgrupos de la muestra, así como la aplicación de criterios de significación estadística entre esas eventuales diferencias. En nuestro caso, por ejemplo, no evidencian diferencias significativas para ninguno de los indicadores en función del género o del centro de procedencia; pero consideramos que su uso puede llegar a ser provechoso cuando la herramienta se generalice y la base de datos a que dé lugar contenga aportes heterogéneos. Por otro lado, la herramienta también nos permite rastrear las posibles correlaciones entre diferentes variables; si bien en nuestro caso estas no resultan especialmente interesantes, pues solo son significativas aquellas fácilmente previsibles (la correlación, por ejemplo, entre el Índice de competencia digital general y cada uno de los subíndices). 
En nuestro proceso de análisis, también podemos proceder al recuento de los promedios que se obtienen para cada uno de los ítems del cuestionario, lo cual nos puede proveer de una interesante radiografía de la competencia digital de nuestros informantes. Así lo vemos, por ejemplo, en las dos tablas siguientes. En la primera, comprobamos cuáles son los ítems en los que los informantes se han manifestado más convencidos de su propia competencia: su comprensión del funcionamiento de los entornos de trabajo colaborativo, los procedimientos para la elaboración de documentos o la comunicación con sus propios compañeros por medio de las TIC, por ejemplo, con promedios iguales o superiores a 4 :

TABLA 2

PROMEDIOS MÁXIMOS DE COMPETENCIA DIGITAL

\begin{tabular}{|l|c|c|}
\hline & Media & Desv. Típ. \\
\hline Toma y descarga de fotografías & 3,38 &, 855 \\
\hline Uso del procesador de textos & 3,29 &, 707 \\
\hline Uso de programas de presentaciones & 3,20 &, 728 \\
\hline Manejo básico del ordenador & 3,19 &, 607 \\
\hline Audición de música & 3,16 & 1,028 \\
\hline
\end{tabular}

Por su parte, nuestros informantes han sido especialmente críticos por lo que respecta a su competencia digital en términos de uso de buscadores, análisis de la información encontrada o participación en redes sociales durante su proceso de enseñanza-aprendizaje, como se refleja en la siguiente tabla:

TABLA 3

PROMEDIOS MÍNIMOS DE COMPETENCIA DIGITAL

\begin{tabular}{|l|c|c|}
\hline & Media & Desv. Típ. \\
\hline Escaneo de documentos & 2,49 & 1,043 \\
\hline Generación de gráficos & 2,48 &, 909 \\
\hline Capturas de pantalla & 2,45 & 1,039 \\
\hline Consulta de mapas & 2,36 &, 802 \\
\hline Consulta y uso de tutoriales & 2,34 &, 895 \\
\hline
\end{tabular}

Por lo que respecta al análisis de la fiabilidad, con los primeros datos de este pilotaje hemos podido calcular el grado de fiabilidad de la herramienta por diferentes procedimientos, entre los cuales presentamos los siguientes.

Según el modelo Alfa de Cronbach para el análisis de la coherencia interna, obtenemos una fiabilidad de 0,908. Como Señala García (2006), la mayoría de los expertos están de acuerdo en exigir un mínimo de 0,60-0,70 cuando el propósito de los estudios es la investigación (en oposición al valor mínimo de 0,95 ) cuando de las decisiones que se 
deriven de un estudio se derivan modificaciones sobre la vida de las personas; por tanto, nos hallamos ante un valor más que suficiente para aceptar el uso de INCOTIC-ESO.

Asimismo, esta aseveración se ve confirmada por otros valores, como los del análisis de mitades, que arroja un coeficiente de Spearman-Brown de 0,831 para el constructor (cuando el umbral es de 0,5 para la fiabilización de instrumentos de medida) y una coherencia interna de 0,914 y 0,732 para cada una de las partes.

Tras la validación de la herramienta por medio de los procedimientos anteriormente explicados, consideramos que estos dos, entre los demás posibles, nos podían ayudar mejor a fiabilizar la herramienta. Si nuestros expertos, por medio de las escalas de pertinencia y univocidad habían señalado ya la armonía teórica del constructo, nos interesaba especialmente conocer cómo se comportaba la herramienta por lo que respecta a su coherencia estadística interna, para lo cual el cálculo de la alfa de Cronbach y el coeficiente de Spearman-Brown son dos de los indicadores más comúnmente utilizados (Glass 1989).

\section{LA COMPETENCIA DIGITALY LA PLANIFICACIÓN DE SU DOCENCIA}

Al finalizar el proceso de creación, validación y fiabilización de la herramienta con la que procederemos a autoevaluar la competencia digital de los estudiantes de los primeros cursos de Educación Secundaria Obligatoria, creemos que hemos conseguido no solo una herramienta aplicable a nuestra contexto más inmediato, el programa $E d u C A T$ $1 \times 1$ de Cataluña, sino que es posible utilizarla en cualquier contexto educativo análogo.

Como decíamos en los primeros apartados, las habilidades digitales han entrado a formar parte del conjunto de destrezas no terminales o específicas cuyo conocimiento deberá acreditar el alumnado en el momento de obtener su título de Educación Secundaria Obligatoria. Sin embargo, podemos afirmar que, en general, no se pretende que las TIC constituyan materia de estudio en sí mismas (si bien es cierto que en gran medida reciben especial atención desde el área de Tecnología, ya sea en esta asignatura o en las obligatorias u optativas de Informática). Bien al contrario, se parte de la incorporación de esta competencia a cualquiera de las materias y/o asignaturas obligatorias de la educación secundaria obligatoria: es el salto, como sabemos, desde las TIC (Tecnologías de la Información y la Comunicación) a las TAC (Tecnologías del Aprendizaje y el Conocimiento) (Vivancos 2008).

En este sentido, debemos tener en cuenta que, en el caso de esta competencia, el alumnado puede que ya tenga una parte muy importante adquirida cuando inicia la ESO, bien gracias a lo aprendido en Educación Primaria, bien por su manejo en la vida cotidiana y en su entorno sociofamiliar. Por ello, quizá no tenga mucho sentido invertir muchos recursos específicos en su docencia, si se comprueba que el nivel inicial es suficiente; en cambio, sí puede resultar provechoso favorecer los espacios y las estrategias para que el alumnado mejore su nivel de adquisición o para cubrir las posibles lagunas concretas que se detecten en él.

De ahí se deriva la importancia de tener una herramienta autodiagnóstica que nos facilite información de partida cuando el estudiante inicia sus estudios de ESO. Solo así podremos planificar nuestra intervención formativa para ayudarle a garantizar el nivel requerido por la legislación educativa. Una vez analizados los datos y la información 


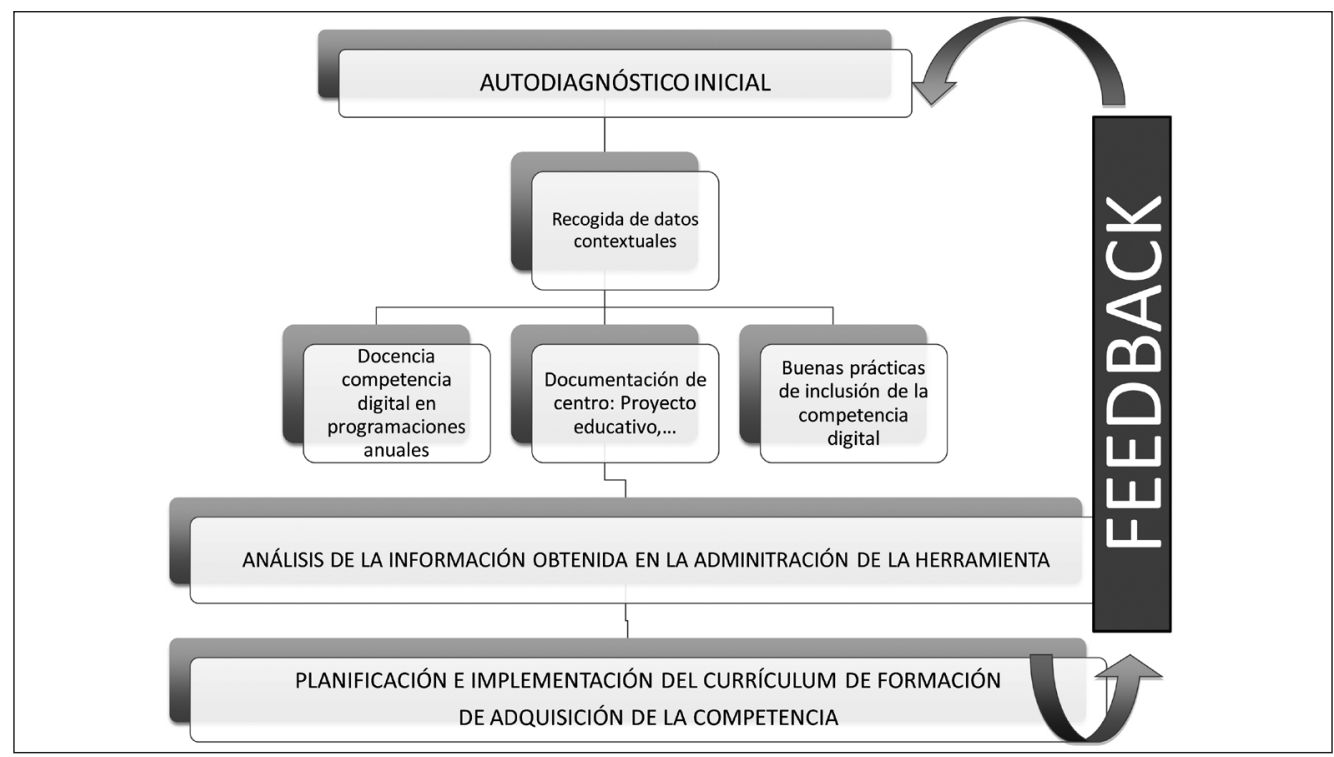

IMAGEN 4

PROPUESTA DE PLANIFICACIÓN DE LA DOCENCIA DE LA COMPETENCIA DIGITAL

que nos facilita la herramienta, proponemos una aproximación a lo que creemos que debe ser la planificación de la docencia de la competencia digital, con independencia de que estime necesario dedicarle un tiempo específico a ello o no. A continuación presentamos la propuesta de manera sintética.

Como vemos en la Imagen 4, plantearnos la planificación de la docencia de la competencia nuclear digital a partir de un autodiagnóstico inicial para poder determinar de manera exacta el nivel de partida teniendo siempre en cuenta que como es la propia percepción del sujeto es posible que tengamos que matizarla. Del mismo modo, será necesario realizar una recogida de datos contextuales con tres finalidades:

- Recabar información acerca de cómo se realiza la docencia de la competencia digital en las programaciones anuales, cuando así sea.

- Analizar cómo se concibe específicamente en la documentación normativa del centro (Proyecto Educativo).

- Seleccionar información que sirva de guía y de ejemplo a la hora de aplicar la competencia digital a las características y contenidos específicos de cada centro.

Tras el análisis de toda esta información y de los datos que nos ha proporcionado la administración de la herramienta INCOTIC-ESO al alumnado, ya estamos en condiciones de planificar e implementar una parte del currículum de formación que tenga como objetivo fundamental cimentar y garantizar el proceso de adquisición de la competencia. Este proceso, por supuesto, no quedará del todo cerrado y evaluado hasta que certifiquemos el nivel de adquisición de la competencia al final de proceso de formación (en este caso, en el momento de la titulación de Graduado en ESO, al final del $4 .^{\circ}$ curso). Esta información nos debe servir no sólo para establecer el nivel de 
salida, sino también como feed-back del proceso de formación para poder mejorarlo o modificarlo en función de aquellos aspectos que no se hayan desarrollado como estaba previsto.

\section{UNA BREVE REFLEXIÓN FINAL}

Sin duda, INCOTIC-ESO es sólo la primera de las múltiples acciones que debemos realizar para asegurar que nuestro alumnado, al finalizar la Educación Secundaria Obligatoria, ha adquirido los niveles suficientes de la competencia digital que debemos exigir a un ciudadano del siglo xxi y que requieren sus estudios superiores, ya profesionales, ya orientados a la Universidad.

Con todo, disponer de esa herramienta - ya validada y fiabilizada, en una primera fase-, al alcance cualquier otra administración educativa que esté en nuestras mismas circunstancias, puede serle de utilidad para planificar, de manera contextualizada en función de los grupos de incidencia, la docencia para la adquisición de esta competencia por parte de los estudiantes de ESO. Y ello, desde una perspectiva transversal del currículum y en función de las características de los estudiantes concretos con los que se tenga que trabajar. INCOTIC-ESO, en última instancia, favorece que podamos incidir directamente en aquellos ámbitos en los que los grupos de estudiantes presenten un menor nivel de competencia.

El autodiagnóstico previo de la competencia en uso de las TIC de nuestro alumnado al iniciar sus estudios secundarios obligatorios nos permite no sólo hacer una planificación «a medida» del proceso de formación, sino también realizar un proceso de seguimiento y de evaluación final que nos facilite determinar el nivel de mejora en términos de aprendizaje, a la vez que garantizar que les estamos dotando de herramientas y estrategias para conseguir ser ciudadanos y estudiantes competentes en la sociedad del conocimiento. INCOTIC-ESO, por, supone la puesta en común con toda la comunidad educativa de nuestro modo de afrontar uno de los retos de la Sociedad del Conocimiento: la incorporación de los contenidos transversales, en general, y de la competencia digital, en particular.

\section{REFERENCIAS}

Aguaded, J. I., Guzmán, M. D., \& Pavón, I. (2010). Convergencia europea y TIC. En AA.VV., Actas del VI Congreso Internacional Virtual de Educación. En prensa.

Bullen, M. et al. (2009). The Net Generation in Higher Education: Rhetoric and Reality. International Journal of Excellence in E-Learning, 2 (1). Recuperado de http://journals. hbmeu.ae/Pages/Articles.aspx?AID=91\&IID=21

Cela, J. M., \& Gisbert, M. (2008). La URV cap a l'EEES. Tarragona, España: Publicacions de la Universitat Rovira i Virgili.

De Pablos, J. (2007). El cambio metodológico en el Espacio Europeo de Educación Superior. Revista Iberoamericana de Educación a Distancia, 10 (2), 15-44.

Decret 89/2009, de 9 de juny, pel qual es regula l'acreditació de competències en tecnologies de la informació i la comunicació (ACTIC). Diari Oficial de la Generalitat de Catalunya de 11 de junio de 2009. 
Diario Oficial de la Unión Europea [DOUE] (2006). Recomendación del Parlamento Europeo y del Consejo de 18 de diciembre de 2006 sobre competencias clave para el aprendizaje permanente. Bruselas, Bélgica: Autor.

Esteve, F. (2009). Bolonia y las TIC: De la docencia 1.0 al aprendizaje 2.0. La Cuestión Universitaria, 5, 59-68.

García Cadena, C. H. (2006). La medición en las ciencias sociales y en psicología. En R. Landeros Hernández \& M.T. González Ramírez (Comps.), Estadística con SPSS y metodología de la investigación (pp. 139-166). México: Trillas.

Generalitat de Catalunya (2010). Continguts de l'ACTIC. Recuperado de http://www20.gencat.cat/docs/actic/01\%20Informacio/Documents/Arxius/Continguts\%20ACTIC_nou.pdf

Gisbert Cervera, M., Espuny Vidal, C., \& González Martínez, J. (2011). INCOTIC. Una herramienta para la @utoevaluación diagnóstica de la competencia digital en la universidad. Revista de Currículum y Formación del Profesorado, 15 (1), 75-90.

González Martínez, J., Espuny Vidal, C., \& Gisbert Cervera, C. (2010a). La evaluacion de la competencia nuclear digital en los nuevos grados del EEES. Revista d'Innovació Educativa, 4, 13-20.

González Martínez, J. et al. (2010b). La Escuela 2.0. Retos de organización y cambio de roles. En A. Manzanares Moya (Ed.), Organizar y dirigir en la complejidad. Instituciones Educativas en evolución (pp. 735-774). Madrid, España: Wolters Kluewer.

Glass, G. V. (1989). Métodos estadísticos aplicados a las ciencias sociales. México: Prentice Hall Hispanoamericana.

Larraz, V. (2011). La competència digital a la Universitat [Documento pilocopiado]. Universitat d'Andorra, Andorra.

Lleixà, M. (2008). La tutoria virtual i la inserció laboral dels professionals d'infermeria (Tesis Doctoral). Recuperado de http://www.tesisenred.net/handle/10803/8940

Marqués, L. (2006). CREDEFIS: Una experiencia de "Blended Learning" en el ámbito de la Educación Física (Trabajo de investigación para la obtención del Diploma de Estudios Avanzados). Universidad Rovira y Virgili, Tarragona (España).

Oblinger, D. G., \& Oblinger, J. L. [Eds.] (2005). Educating the Net Generation. Washington, DC: EDUCAUSE.

Secretaría de Telecomunicacions i Societat de la Informació (2004). Enquesta a les llars sobre equipament $i$ ús de les Tecnologies de la Informació i la Comunicació (TIC) a Catalunya - 2003. Recuperado de http://www10.gencat.net/dursi/pdf/si/observatori/ documents_STSI/informe_TIC_\%201lars_\%20i_\%20individus\%202003.pdf

Storey, M. A., Phillips, B., Maczewski, M., \& Wang, M. (2002). Evaluating the usability of Web-based learning tools. Educational Technology E Society, 5 (3). Recuperado de http://www.ifets.info/journals/5_3/storey.html

Universitat Rovira i Virgili (2009). Competències transversals. Guia per treballar i avaluar les competències transversals a les titulacions de Grau. Tarragona, España: Autores.

Vivancos, J. (2008). Tratamiento d la información y competencia digital. Madrid, España: Alianza Editorial.

Fecha de recepción: 28 de febrero de 2011.

Fecha de revisión: 28 de febrero de 2011.

Fecha de aceptación: 28 de agosto de 2011. 